\title{
Análise da Revisão Cochrane: Antibioticoterapia na Otite Média Aguda da Criança. Cochrane Database Syst Rev. 2013;1:CD000219.
}

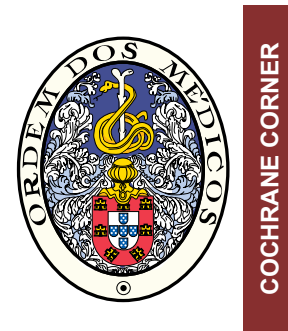

\author{
Analysis of the Cochrane Review: Antibiotics for Acute Otitis Media in Children. \\ Cochrane Database Syst Rev. 2013;1:CD000219.
}

\author{
Sara PINTO1, João COSTA ${ }^{2,3,4}$, António VAZ CARNEIRO ${ }^{3,4}$, Ricardo FERNANDES ${ }^{1,2}$ \\ Acta Med Port 2013 Nov-Dec;26(6):633-636
}

Palavras-chave: Otite Média Aguda; Criança; Antibióticos; Revisão Sistemática.

Keywords: Otitis Media; Child; Anti-Bacterial Agents; Systematic Reviews.

\section{RESUMO}

A otite média aguda é uma das infeções mais frequentes na criança e um dos principais motivos de prescrição antibiótica. Neste artigo, avaliamos e comentamos a revisão sistemática da Cochrane 'Antibiotics for acute otitis media in children', cujos objetivos eram avaliar a eficácia e a segurança da antibioticoterapia na otite média aguda da criança e identificar subgrupos que mais beneficiem deste tratamento. Esta revisão mostrou resolução espontânea de otite média aguda na maioria dos doentes ( $82 \%)$ e um efeito favorável mas modesto da antibioticoterapia, nomeadamente na resolução da otalgia (number needed to treat to benefit: 20) e na redução das perfurações timpânicas e da otite média aguda contralateral. Observou-se maior risco de efeitos adversos (vómitos, diarreia ou exantema) no grupo da antibioticoterapia (number needed to treat to harm: 14). Assim, parece justificar-se uma atitude de vigilância na maioria das crianças durante 48-72h, sem prescrição antibiótica inicial. Uma meta-análise complementar identificou maior benefício da antibioticoterapia em crianças com otorreia associada a otite média aguda e em crianças menores de 2 anos com otite média aguda bilateral.

\section{ABSTRACT}

Acute otitis media is one of the most common infections in children and one of the leading causes for antibiotic prescription. In this paper, we assess and comment the Cochrane systematic review 'Antibiotics for acute otitis media in children', which aimed at assessing the efficacy and safety of antibiotics for acute otitis media in children and identifying subgroups of children who might benefit more than others from antibiotic treatment. This review showed spontaneous resolution of acute otitis media in most children (82\%) and a favorable but modest effect of antibiotics, namely in pain control (number needed to treat to benefit: 20), reduction of tympanic membrane perforations and reduction of contralateral acute otitis media. Adverse effects such as vomiting, diarrhea or rash were more common in the antibiotic group (number needed to treat to harm: 14). Thus, for most children, an expectant observational approach during 48-72h without immediate antibiotic prescription seems justified. An additional meta-analysis found that antibiotics appear to be most useful in children with both acute otitis media and otorrhoea and children under two years of age with bilateral acute otitis media.

\section{INTRODUÇÃO}

A otite média aguda (OMA) é uma das infeções mais frequentes em idade pediátrica e um dos principais motivos de prescrição antibiótica nesta faixa etária. ${ }^{1}$ Embora a antibioticoterapia esteja claramente indicada nalguns grupos de doentes com OMA, a resolução espontânea na maioria dos casos leva a que se questione a utilização indiscriminada dos antibióticos. ${ }^{2}$ Assim, poderá justificar-se a adoção duma atitude de vigilância sem prescrição antibiótica inicial na maioria dos doentes.

\section{OBJETIVO DO ESTUDO}

Avaliar a eficácia e a segurança da antibioticoterapia na OMA da criança, assim como identificar subgrupos que mais beneficiem deste tratamento.

\section{MÉTODOS}

Trata-se de uma revisão sistemática de ensaios clínicos controlados e aleatorizados que compararam antibioticoterapia versus placebo ou antibioticoterapia imediata versus atitude de vigilância (com ou sem prescrição suspensa de antibiótico) em crianças com diagnóstico de OMA. Escolheram-se outcomes relevantes para os doentes: o outcome primário foi a presença de otalgia em diferentes momentos da evolução da doença (até ao $7^{\circ} \mathrm{dia}$ ) e os outcomes secundários foram a presença de complicações agudas (como perfuração timpânica, mastoidite, meningite), desenvolvimento de otite contralateral (se inicialmente unilateral), recorrências de OMA, presença de défice auditivo (timpanometria às 4-6 semanas e 3 meses) e ocorrência de efeitos adversos relacionados com a antibioticoterapia (vómitos, diarreia, exantema).

1. Departamento de Pediatria. Hospital de Santa Maria. Centro Hospitalar Lisboa Norte. Lisboa. Portugal.

2. Unidade de Farmacologia Clínica. Instituto de Medicina Molecular. Faculdade de Medicina. Universidade de Lisboa. Lisboa. Portugal.

3. Centro de Estudos de Medicina Baseada na Evidência. Faculdade Medicina da Universidade de Lisboa. Lisboa. Portugal.

4. Centro Colaborador Português da Rede Cochrane Iberoamericana. Lisboa. Portugal.

Recebido: 05 de Dezembro de 2013 - Aceite: 06 de Dezembro de 2013 | Copyright $\odot$ Ordem dos Médicos 2013 


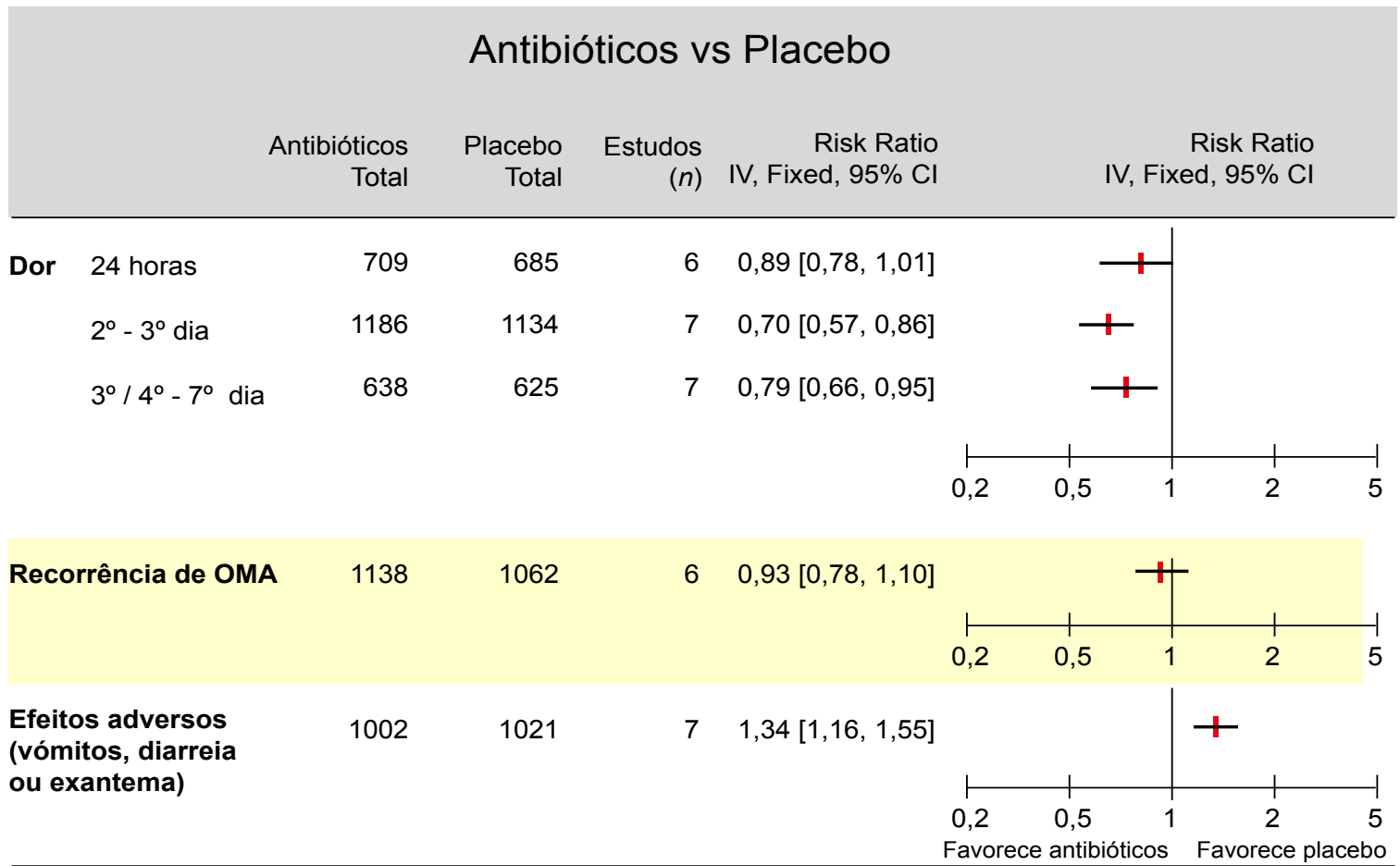

Figura 1 - Comparação dos outcomes dor, recorrência de OMA e efeitos adversos nos ensaios antibioticoterapia versus placebo (esquerda) e ensaios antibioticoterapia imediata versus atitude de vigilância (direita).

* $4^{\circ}-7^{\circ}$ dia nos ensaios antibioticoterapia versus placebo e $3^{\circ}-7^{\circ}$ dia nos ensaios antibioticoterapia imediata versus atitude de vigilância Adaptado de Venekamp RP, Sanders S, Glasziou PP, Del Mar CB, Rovers MM. Antibiotics for acute otitis media in children. Cochrane Database of Systematic Reviews 2013, Issue 1. Art. No.: CD000219. DOI: 10.1002/14651858.CD000219.pub3.

\section{RESULTADOS}

Foram analisados 11 ensaios que compararam antibioticoterapia versus placebo, tendo sido incluídas 3317 crianças menores de 12 anos. A antibioticoterapia mostrou reduzir a otalgia entre o $2^{\circ}$ e $\circ 3^{\circ}$ dia (risco relativo [RR] 0,70 ; intervalo de confiança [IC] 95\% 0,57-0,86) e entre o $4^{\circ}$ e o $7^{\circ}$ dia (RR 0,79; IC 95\% 0,66-0,95) - estes resultados do outcome primário tiveram reduzida heterogeneidade $\left(I^{2}=0-29 \%\right)$. Houve também redução do risco de perfuração timpânica (RR 0,37; IC 95\% 0,18-0,76) e de OMA contralateral (RR 0,49; IC 95\% 0,25-0,95), mas maior risco de efeitos adversos, incluindo vómitos, diarreia ou exantema (RR 1,34; IC 95\% 1,16-1,55) - Fig. 1. Comparativamente ao placebo, não se observou efeito significativo da antibioticoterapia na redução da otalgia às 24 horas de doença, na redução da taxa de recorrência de OMA ou na redução do número de crianças com défice auditivo às 4-6 semanas ou aos 3 meses. As complicações graves, como mastoidite e meningite, foram raras em ambos os grupos (apenas 3 casos na população incluída). Foram também analisados 4 ensaios que compararam antibioticoterapia imediata versus atitude de vigilância (com ou sem prescrição suspensa de antibiótico), tendo sido incluídas 1149 crianças menores de 15 anos. Não se observou diferença estatisticamente significativa na presença de otalgia entre $\circ 3^{\circ}$ e o $7^{\circ} \mathrm{dia}$ (resultados com heterogeneidade substancial, $I^{2}=71 \%$ ), nem no aparecimento de perfuração timpânica ou recorrência de OMA. Não ocorreram complicações graves em nenhum dos grupos. A antibioticoterapia imediata esteve associada a maior ocorrência de efeitos adversos (RR 1,71; IC 95\% 1,24-2,36) - Fig. 1. Uma meta-análise efetuada pelos mesmos autores da revisão Cochrane utilizou dados individuais de doentes de alguns dos ensaios incluídos (6 estudos, 1647 crianças), o que permitiu estudar com maior rigor o benefício e risco em determinados subgrupos. ${ }^{3}$ Esta meta-análise complementar mostrou maior benefício da antibioticoterapia na redução de OMA prolongada (dor, febre ou ambos entre o $3^{\circ}$ e o $7^{\circ}$ dia) nas crianças com otorreia associada a OMA e nas crianças menores de 2 anos de idade com OMA bilateral.

\section{CONCLUSÕES}

A antibioticoterapia está associada a uma ligeira redução no número de crianças com otalgia do $2^{\circ}$ ao $7^{\circ}$ dia de doença, no número de perfurações timpânicas e de OMA contralateral. No entanto, a maioria dos episódios de OMA remite espontaneamente pelo que o risco de efeitos adversos associados ao antibiótico devem ser considerados. Crianças com otorreia associada a OMA e crianças menores de 2 anos com OMA bilateral são as que mais beneficiam de tratamento antibiótico. Para as restantes crianças com doença ligeira, pode ser considerado um período inicial de vigilância.

\section{COMENTÁRIOS}

O papel da antibioticoterapia na OMA da criança tem sido extensamente discutido nos últimos anos. $O$ facto da etiologia da OMA ser predominantemente bacteriana sus- 
Antibióticos vs Vigilância

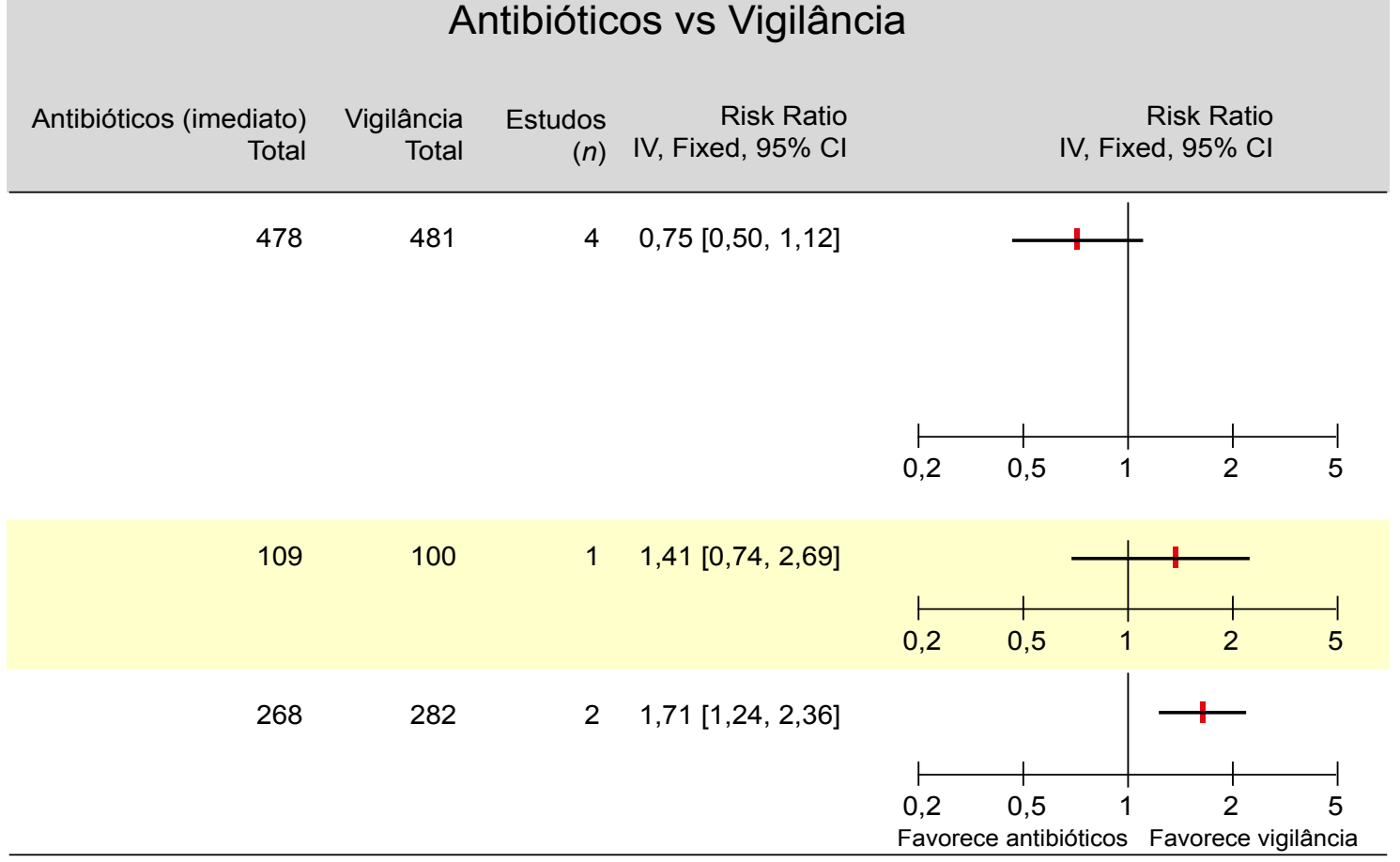

Figura 1 (continuação) - Comparação dos outcomes dor, recorrência de OMA e efeitos adversos nos ensaios antibioticoterapia versus placebo (esquerda) e ensaios antibioticoterapia imediata versus atitude de vigilância (direita).

* $4^{\circ}-7^{\circ}$ dia nos ensaios antibioticoterapia versus placebo e $3^{\circ}-7^{\circ}$ dia nos ensaios antibioticoterapia imediata versus atitude de vigilância Adaptado de Venekamp RP, Sanders S, Glasziou PP, Del Mar CB, Rovers MM. Antibiotics for acute otitis media in children. Cochrane Database of Systematic Reviews 2013, Issue 1. Art. No.: CD000219. DOI: 10.1002/14651858.CD000219.pub3.

tenta o uso de antibióticos de um ponto de vista teórico, mas eventuais benefícios clínicos devem ser ponderados face à história natural da doença, aos efeitos adversos da intervenção e aos riscos microbiológicos conhecidos relacionados com as resistências induzidas por práticas desadequadas de antibioticoterapia.

Esta revisão mostra um efeito favorável mas modesto da antibioticoterapia. A resolução foi espontânea na maioria dos casos $\left(82 \%\right.$ entre o $2^{\circ}$ e o $\left.7^{\circ} \mathrm{dia}\right)$ e o uso de antibióticos contribuiu para a redução da otalgia neste período em apenas um terço dos doentes. Por outras palavras, 20 crianças têm de ser tratadas com antibiótico para prevenir otalgia numa criança (number needed to treat to benefit). Por outro lado, a antibioticoterapia esteve associada a maior risco de efeitos adversos (nesta revisão, por cada 14 crianças tratadas com antibiótico, uma sofreu um efeito adverso number needed to treat to harm), a que acresce o risco de aumento das resistências bacterianas ${ }^{4}$ e dos custos.

Assim, o balanço entre o benefício modesto da antibioticoterapia e os riscos associados suporta a adoção de uma atitude de observação na maioria das crianças. Esta consiste num período de vigilância de 48 a 72 horas apenas com tratamento sintomático e com instituição posterior de antibioticoterapia se o estado clínico se deteriora ou não melhora. Nesta revisão, não houve diferença nos outcomes dos doentes submetidos a atitude de observação comparativamente aos que efetuaram antibiótico imediato, exceto no aumento dos efeitos adversos nestes.

Devemos realçar que a aplicação clínica destes dados deve ter em conta os subgrupos que foram identificados como podendo beneficiar mais de antibiótico ${ }^{3}$ e os doentes com maior risco de complicações (por exemplo com quadro clínico grave inicial), para os quais os benefícios podem superar os riscos. De referir igualmente que esta revisão não analisou em detalhe o esquema de antibioticoterapia escolhido, nomeadamente o antibiótico específico, a dose e a duração do tratamento.

A adoção de uma atitude de vigilância ou de uma prescrição antibiótica inicial apenas em doentes selecionados tem sido incluída nas orientações clínicas de vários países. Em Portugal, a norma clínica da Direção-Geral de Saúde 'Diagnóstico e Tratamento da Otite Média Aguda na Idade Pediátrica' de $2012^{5}$ recomenda a prescrição imediata de antibiótico em (1) lactentes menores de 6 meses de idade ou em (2) quadro clínico grave ou (3) presença de otorreia ou (4) otite bilateral (em menores de 2 anos de idade). Nos restantes doentes é aconselhado um período de vigilância de 48-72 horas, assegurando-se que há possibilidade de reavaliação clínica, ou em alternativa, uso de prescrição retardada para início de tratamento pelos cuidadores se houver persistência ou agravamento de sintomas. Recomendações similares foram emitidas este ano pela American Academy of Pediatrics. ${ }^{6}$ Estas recomendações e os resultados dos vários ensaios pressupõem sempre um diagnóstico rigoroso de OMA. Este é um passo primordial na abordagem destes doentes, para que o tratamento antibiótico possa ser dirigido aos que dele possam beneficiar e para evitar o seu uso indevido. 
REFERENCIAS

1. Grijalva CG, Nuorti JP, Griffin MR. Antibiotic prescription rates for acute respiratory tract infections in US ambulatory settings. JAMA. 2009;302:758-66.

2. Rosenfeld RM, Vertrees JE, Carr J, Cipolle RJ, Uden DL, Giebink GS, et al. Clinical efficacy of antimicrobial drugs for acute otitis media: metaanalysis of 5400 children from thirty-three randomized trials. J Pediatr. 1994;124:355-67.

3. Rovers MM, Glasziou P, Appelman CL, Burke P, McCormick DP, Damoiseaux RA, et al. Antibiotics for acute otitis media: a meta-analysis with individual patient data. Lancet. 2006;368:1429-35.
4. Goossens H, Ferech M, Vander Stichele R, Elseviers M, Group EP. Outpatient antibiotic use in Europe and association with resistance: a crossnational database study. Lancet. 2005;365:579-87.

5. Diagnóstico e Tratamento da Otite Média Aguda na Idade Pediátrica. 2012; Norma Clínica da Direção-Geral de Saúde n007/2012. [consultado 2013 Nov 8] Disponível em: http://www.dgs.pt/normas-clinicas.aspx.

6. Lieberthal AS, Carroll AE, Chonmaitree T, Ganiats TG, Hoberman A, Jackson MA, et al. The diagnosis and management of acute otitis media. Pediatrics. 2013 ;131:e964-99.

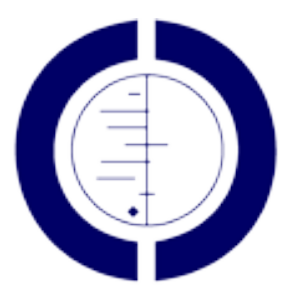

THE COCHRANE COLLABORATION 


\section{Análise da Revisão Cochrane: Antibioticoterapia na Otite Média Aguda da Criança. Cochrane Database Syst Rev. 2013;1:CD000219. Acta Med Port 2013:26:633-636}

Publicado pela Acta Médica Portuguesa, a Revista Científica da Ordem dos Médicos

Av. Almirante Gago Coutinho, 151

1749-084 Lisboa, Portugal.

Tel: +351218428215

E-mail: submissao@actamedicaportuguesa.com

www.actamedicaportuguesa.com

ISSN:0870-399X | e-ISSN: 1646-0758

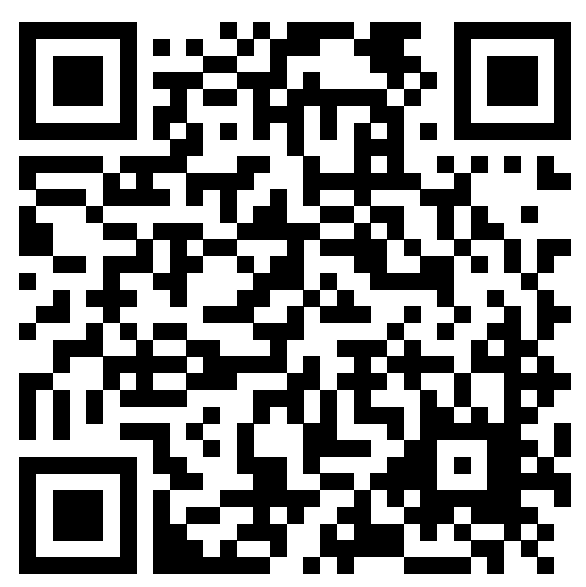

